\title{
Commentary: Getting to the heart of postoperative atrial fibrillation after cardiac surgery
}

\author{
Rachel Eikelboom, MD, ${ }^{a}$ and Rakesh C. Arora, MD, PhD, FRCSC ${ }^{a, b}$
}

\footnotetext{
From the ${ }^{\mathrm{a} D e p a r t m e n t}$ of Surgery, Max Rady College of Medicine, Winnipeg, Manitoba, Canada; and ${ }^{\mathrm{b} C a r d i a c}$ Sciences Program, St Boniface Hospital, Winnipeg, Manitoba, Canada.

Disclosures: Arora has received an unrestricted educational grant from Pfizer Canada Inc and honoraria from Mallinckrodt Pharmaceuticals for work unrelated to this article. Eikelboom has nothing to disclose with regard to commercial support.

Received for publication March 7, 2019; accepted for publication March 8, 2019; available ahead of print April $23,2019$.

Address for reprints: Rakesh C. Arora, MD, PhD, FRCSC, Section of Cardiac Surgery, Department of Surgery, University of Manitoba, St Boniface General Hospital, CR3015, 369 Tache Ave, Winnipeg, Manitoba R2H 2A7, Canada (E-mail: rakeshcarora@gmail.com).

J Thorac Cardiovasc Surg 2020;159:524-10

$0022-5223 / \$ 36.00$

Copyright (c) 2019 by The American Association for Thoracic Surgery

https://doi.org/10.1016/j.jtcvs.2019.03.039
}

Postoperative atrial fibrillation (POAF) is associated with prolonged hospital stay, greater health care costs, and increased mortality after cardiac surgery. ${ }^{1}$ Effective prophylactic measures, such as corticosteroids and antiarrhythmics, are not widely used, perhaps because of concerns about side effects and costs. ${ }^{2}$ A clinical prediction model for POAF might encourage targeted use in higher-risk patients, but development of such a model requires improved understanding of POAF pathophysiology and risk factors.

In this issue of the Journal, Bening and colleagues ${ }^{3}$ address an important gap in the literature by investigating predictors of POAF. Casting a wide net, they examine atrial muscle fiber function, echocardiographic measurements of heart chamber size and function, and biochemical markers of inflammation.

Bening and colleagues ${ }^{3}$ demonstrate an association between impaired left atrial contractility and occurrence of POAF. Measurement of left atrial contractility is a laborintensive process of harvesting atrial tissue intraoperatively, preparing skinned myocardial fibers, exposing them to increasing concentrations of calcium, and measuring their contractile force. Results are only available postoperatively, whereas evolving evidence suggests POAF prophylaxis should start preoperatively or intraoperatively. ${ }^{4,5}$ The clinical applicability of this unwieldly process is therefore limited. The authors ${ }^{3}$ also report that echocardiographic evidence of increased right atrial size and depressed right ventricular function may predict POAF, which has not been previously reported in the literature and merits further exploration.

Bening and colleagues ${ }^{3}$ suggest that patients with impaired left atrial contractility and echocardiographic evidence of right heart dysfunction may benefit from postoperative cardiac monitoring and magnesium and potassium supplementation. At our institution, and we suspect in many other centers, these measures are routinely used for POAF prophylaxis. ${ }^{2}$

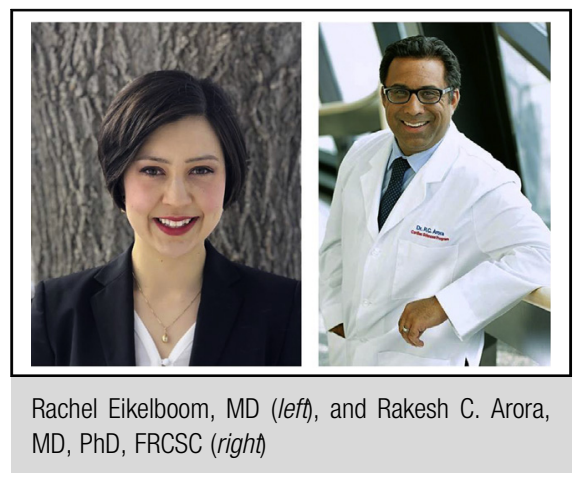

Central Message

Much mechanistic study is yet required to develop potential predictors of postoperative atrial fibrillation into a clinically useful risk prediction model.

See Article page 515

all postoperative cardiac surgical patients. Although relatively low risk and low cost, they are also ineffective for

Clinical prediction models must discriminate between patients who will experience an event and those who will not. Clinicians can use such models to weigh the risks of an event, such as POAF, against the risks of interventions, such as corticosteroids and antiarrhythmics. ${ }^{6}$ Bening and colleagues $^{3}$ unfortunately do not report cutoff values for atrial contractility or echocardiographic parameters, which would permit discrimination between higher and lower risk patients. Neither do they describe the degree of risk conferred by the presence of each predictor. Future work should examine these predictors in larger populations.

Another area for future work is the mechanistic pathophysiology of POAF. Despite previous research suggesting links between POAF and inflammation, ${ }^{7}$ why were none of the inflammatory biomarkers measured in the study of Bening and colleagues ${ }^{3}$ predictive of POAF? By what mechanism does right heart dysfunction increase risk of POAF?

Bening and colleagues ${ }^{3}$ have provided both impetus and direction for exciting future research into the mechanisms of and risk factors for POAF after cardiac surgery. Ultimately, their research moves us closer to a clinical risk prediction model for POAF, which would allow individualized POAF risk management. 


\section{References}

1. Lowres N, Mulcahy G, Jin K, Gallagher R, Neubeck L, Freedman B. Incidence of postoperative atrial fibrillation recurrence in patients discharged in sinus rhythm after cardiac surgery: a systematic review and meta-analysis. Interact Cardiovasc Thorac Surg. 2018;26:504-11.

2. Turagam MK, Downey FX, Kress DC, Sra J, Tajik AJ, Jahangir A. Pharmacological strategies for prevention of postoperative atrial fibrillation. Expert Rev Clin Pharmacol. 2015;8:233-50.

3. Bening C, Mazalu EA, Yaqub J, Alhussini K, Glanowski M, Kottmann T, et al. Atrial contractility and fibrotic biomarkers are associated with atrial fibrillation after elective coronary artery bypass grafting. J Thorac Cardiovasc Surg. 2020;159: 515-23.

4. Chatterjee S, Sardar P, Mukherjee D, Lichstein E, Aikat S. Timing and route of amiodarone for prevention of postoperative atrial fibrillation after cardiac surgery:

a network regression meta-analysis. Pacing Clin Electrophysiol. 2013;36: 1017-23.

5. Arsenault KA, Yusuf AM, Crystal E, Healey JS, Morillo CA, Nair GM et al. Interventions for preventing post-operative atrial fibrillation in patients undergoing heart surgery. Cochrane Database Syst Rev 2013;1: CD003611.

6. Alba AC, Agoritsas T, Walsh M, Hanna S, Iorio A, Devereaux PJ, et al. Discrimination and calibration of clinical prediction models: users' guides to the medical literature. JAMA. 2017;318:1377-84.

7. Narducci ML, Pelargonio G, Rio T, Leo M, Di Monaco A, Musaico F, et al. Predictors of postoperative atrial fibrillation in patients with coronary artery disease undergoing cardiopulmonary bypass: a possible role for myocardial ischemia and atrial inflammation. J Cardiothorac Vasc Anesth. 2014;28:512-9. 